\title{
ARTÍCULOS
}

\section{POLÍTICAS PÚBLICAS DE DESARROLLO DE LA INDUSTRIA DE CONTENIDOS DIGITALES: UNA PROSPECTIVA INTERNACIONAL}

Alberto Urueña-López y Antonio Hidalgo-Nuchera
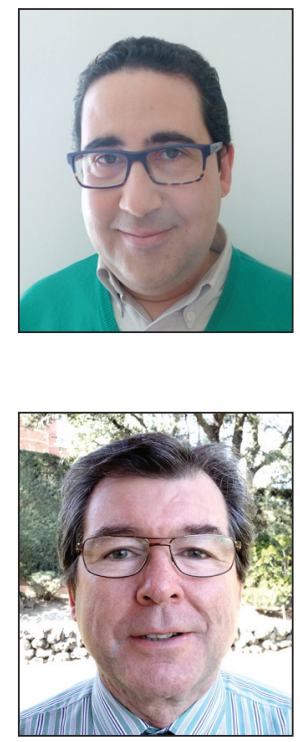

Alberto Urueña-López es subdirector adjunto del Observatorio Nacional de las Telecomunicaciones y de la Sociedad de la Información de Red.es. Doctor ingeniero industrial en el Programa de Economía y Gestión de la Innovación e ingeniero de telecomunicación por la Universidad Politécnica de Madrid (UPM), executive MBA por el Instituto de Empresa y máster en economía de las telecomunicaciones por la UNED. Investigador del Grupo Innopro de la UPM, sus líneas de trabajo son el estudio de la sociedad de la información y su impacto en ciudadanos y empresas.

http://orcid.org/0000-0002-1299-2380

alberto.uruena@upm.es

\begin{abstract}
Antonio Hidalgo-Nuchera es catedrático de organización de empresas en la Escuela Técnica Superior de Ingenieros Industriales de la Universidad Politécnica de Madrid (UPM) y director del Grupo de Investigación en Innovación, Propiedad Industrial y Política Tecnológica (Innopro). Ha participado en proyectos financiados por la Comisión Europea y organismos nacionales sobre innovación tecnológica, transferencia de tecnología y desarrollo de políticas tecnológicas. http://orcid.org/0000-0002-3598-9862
\end{abstract}

antonio.hidalgo@upm.es

Universidad Politécnica de Madrid, ETSI Industriales Depto. Ingeniería de Organización, Administración de Empresas y Estadística José Gutiérrez Abascal, 2. 28006 Madrid, España

\section{Resumen}

Se realiza un análisis de políticas públicas para el desarrollo de la industria de contenidos digitales en Alemania, Reino Unido, Francia e Italia (países cercanos al entorno cultural, económico y político de España) e Israel y Estados Unidos (países con un destacado sector de contenidos digitales). Del análisis de las políticas en estos países se han detectado tres líneas de acción: creación y fomento de polos empresariales, búsqueda de nuevas formas de suministrar contenidos a la demanda y programas específicos de internacionalización. Para estas líneas se describen medidas específicas de políticas de los países objeto de la comparativa que, a juicio de los expertos nacionales e internacionales, han tenido impacto en la evolución de la industria de los contenidos digitales de los países seleccionados y que podrían ser de interés para su aplicación en España.

\section{Palabras clave}

Políticas públicas, Industria de contenidos, Contenidos digitales, Análisis de políticas industriales, Impacto de políticas.

\section{Title: Public policies for the development of digital content industry: an international prospective}

\begin{abstract}
This research has been an analysis of public policies for the development of the digital content industry developed in Germany, UK, France and Italy (countries close to our cultural, economic and political) and Israel and the United States (countries with high development of digital content sector). The analysis of policy measures in these countries has identified three lines of action: creating and fostering entrepreneurial centers, finding new ways to deliver content to specific programs demand and internationalization. For each action line describes specific policy measures in the countries under comparison that, according to national and international experts, have had an impact on the development of the digital content industry in selected countries and could be of interest for application to the content sector in Spain.
\end{abstract}

\section{Keywords}

Public policy, Content industry, Digital content, Industrial policy analysis, Policy impact. 
Urueña-López, Alberto; Hidalgo-Nuchera, Antonio (2014). "Políticas públicas de desarrollo de la industria de contenidos digitales: una prospectiva internacional”. El profesional de la información, marzo-abril, v. 23, n. 2, pp. $119-125$.

http://dx.doi.org/10.3145/epi.2014.mar.03

\section{Introducción. Políticas de contenidos digitales en España}

La industria de contenidos digitales en España facturó 8.553 millones de euros en 2011 (Ontsi, 2012), lo que supone el $52,8 \%$ de la facturación total del mercado de contenidos en España, siendo el sector audiovisual el motor de la industria (Ontsi, 2012). El peso de los contenidos digitales frente a los tradicionales continúa en aumento, siendo en 2010 por primera vez cuando los contenidos digitales superaron a los tradicionales (el $53,7 \%$ de las actividades de contenidos y servicios audiovisuales es digital).

La economía de la información y del conocimiento puede llegar a representar un nuevo modelo económico que ayude a superar la crisis actual (Urueña-López; Hidalgo-Nuchera, 2013). En el sector de contenidos digitales, como parte esencial de la nueva economía, se producen todavía incertidumbres sobre planes específicos de negocio para esta industria que sean sostenibles a lo largo del tiempo (Gaptel, 2006). Dado el carácter innovador del sector y su importancia estratégica, en la gran mayoría de los países de nuestro entorno se ha considerado necesario apoyarlo a través de políticas públicas gubernamentales.

\section{Dado el carácter innovador del sector de contenidos y su importancia estratégica es necesario apoyarlo a través de políti- cas públicas gubernamentales}

En esta línea, la Agenda Digital para España aprobada en Consejo de Ministros en febrero de 2013 (Ministerio, 2013a) tiene como objetivos el impulso, producción y distribución de contenidos en internet mediante las siguientes actuaciones:

- plan integral para la industria de contenidos digitales en España;

- adaptación del régimen de derechos de autor -dentro del marco europeo- para la utilización de contenidos digitales;

- simplificación de las condiciones para la reutilización de la información del sector público.

Estas medidas se han desarrollado en un plan detallado de impulso de la economía digital y los contenidos digitales publicado en julio de 2013 (Ministerio, 2013b). El plan propone tres grupos de iniciativas:

- de talento, orientadas a aumentar las competencias y habilidades en el sector de los contenidos digitales;

- de financiación, a través de medidas que promuevan la inversión;

- de carácter industrial, que promuevan el desarrollo del sector industrial de contenidos digitales en España.
Esta investigación se centra en las iniciativas políticas de carácter industrial del sector de contenidos digitales, y tiene como objetivo identificar buenas prácticas en políticas públicas ya desarrolladas en otros países que hayan sido de utilidad, de tal manera que puedan servir como ayuda a la elaboración de políticas públicas en España.

\section{Metodología de la investigación}

Para llevar a cabo la investigación se ha realizado un análisis de políticas públicas basándose en observaciones sobre fenómenos similares en otros países. Utilizando este análisis y la comparación de las políticas públicas en varias zonas geográficas es posible comprender sus especificidades en diferentes contextos económicos, políticos y sociales, así como su evolución a lo largo del tiempo (Bulcourf; Cardozo, 2008).

Como indican Grau y Mateos (2002), el análisis comparado de políticas públicas permite, a través de la búsqueda de variables explicativas, establecer similitudes y diferencias entre políticas similares. Este método es utilizado habitualmente para la medición de los resultados e impactos de políticas públicas (Parsons, 2008).

Bouchard (2000) señala que la selección de los países es esencial para realizar el análisis de las políticas públicas que lleven a un resultado concluyente. Es necesario explicitar el método de elección utilizado para la selección de zonas geográficas que tiene que tener coherencia con los objetivos de la investigación, para que posteriormente puedan explicarse semejanzas y diferencias en las políticas analizadas (Smelser, 2003). En este sentido, el alcance de la investigación se ha circunscrito a países de nuestro entorno geográfico, con similares características políticas, económicas y de adopción y de utilización de nuevas tecnologías que están sometidas a una misma regulación a nivel europeo, siendo elegidos Alemania, Reino Unido, Francia e Italia. Además, el análisis se ha completado con países que tienen un reconocimiento internacional en los contenidos digitales como Israel y Estados Unidos.

\section{Investigación: desarrollo y resultados}

Para cumplir con la metodología planteada, la investigación se ha llevado a cabo en cuatro fases:

\section{Fase 0}

Se realizaron entrevistas a cinco expertos españoles en la industria de contenidos digitales, garantizando su anonimato para que se expresasen libremente. Entre los criterios de selección de los expertos del grupo se eligieron perfiles profesionales con amplia experiencia y de reconocido prestigio en el sector de contenidos digitales, capaces de valorar planes y medidas de desarrollo de la sociedad de la información en general y de contenidos digitales en particular. De esta forma, dos expertos provienen de asociaciones de 
empresas de tecnologías de la información y de contenidos digitales, uno de la administración pública y dos provienen de una firma de consultoría especializada en el mercado de contenidos digitales.

En la entrevista, de carácter semiestructurado, tras una breve introducción sobre el objetivo y fases del estudio, se les pedía que se centrasen en los retos que tenía por delante la industria en los próximos años, pidiéndoles opinión a título estrictamente personal sin que necesariamente fuese coincidente con las de las organizaciones en las que desempeñan su trabajo. Las respuestas más relevantes indicadas por los entrevistados, entre los que hubo un importante grado de coincidencia, fueron:

- superar las dificultades de la internacionalización y salida al exterior;

- incrementar el espíritu emprendedor y ayudar a las empresas sobre todo en las primeras etapas de vida;

- fomentar la colaboración público-privada para buscar nuevas fórmulas de explotar el patrimonio cultural;

- incentivar la creación de polos de competitividad y fomentar la I+D;

- disponer de una regulación en el marco de la Unión Europea que garantice la defensa de los derechos de propiedad intelectual.

\section{Fase 1}

Se han recopilado proyectos y políticas públicas de desarrollo de la industria de los contenidos digitales de los países seleccionados (Alemania, Reino Unido, Francia, Italia, Israel y Estados Unidos). En total se han recogido 127 medidas de 47 fuentes de información, básicamente webs de organismos e instituciones públicas (ministerios, empresas públicas...) que juegan un papel clave en el fomento de la industria de contenidos digitales, tecnológicos y culturales de cada país.

En Italia se encontraron medidas de tipo legislativo de fomento del emprendimiento, más que de desarrollo de políticas públicas de contenidos digitales, por lo que se decidió su eliminación para las siguientes fases de investigación.

Para llevar a cabo la investigación se ha realizado un análisis de políticas públicas basándose en las observaciones sobre fenómenos similares en varios países

\section{Fase 2}

Para el contraste del impacto de las medidas de las políticas públicas recopiladas en la fase 1 se contactó con las principales asociaciones empresariales de los países.

El objetivo de esta fase era encontrar las medidas de políticas públicas de mayor impacto en opinión de la industria de cada país, bien porque a juicio de los entrevistados consideraban que habían tenido un impacto de carácter macroeconómico o social, porque se hubiese realizado un análisis interno de impacto de las medidas o porque, en la experiencia de la asociación, era más favorable a determinado tipo de actuación.

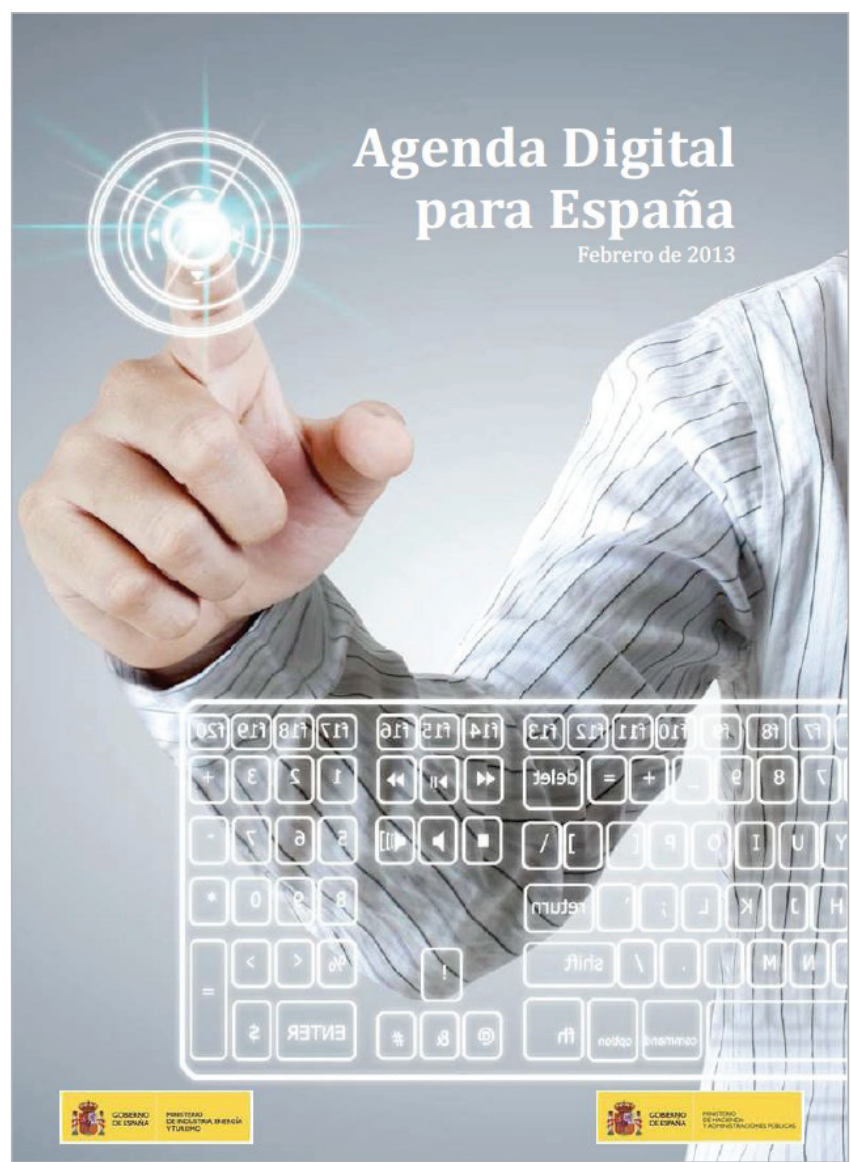

http://www.agendadigital.gob.es

En concreto se contactó en Alemania con asociaciones de empresas como Bitkom, Eco (asociación de la industria alemana de internet) y BVDW (Bundesverband Digitale Wirtschaft, asociación federal de la economía digital). En Reino Unido se contactó con ISPA (Internet Service Providers Association) y Ukita (United Kingdom Information Technology Association). En Francia se solicitó opinión a la Afnet (Association Française de Utilisateurs du Net) y Afdel (Association Française des Editeurs de Logiciels et Solutions Internet). Finalmente en Estados Unidos se contactó con Techamerica y en Israel con Israel Advance Tecnology Industries (ITAA).

La recopilación de la opinión sobre el impacto y oportunidad de las medidas -obtenida a través de entrevistas telefónicas o por correo electrónico a un portavoz de la asociación empresarial a los que se había contactado explicando el estudio y sus objetivos- se ha complementado con informes y análisis públicos sobre el impacto de las iniciativas cuando estaban disponibles (Tech City, 2011). De las 127 medidas recopiladas en la fase 1 se seleccionó un total de 21 de impacto relevante.

\section{Fase 3}

Tras una valoración detallada del contenido de las 21 medidas obtenidas en la fase 2 , se procedió a su clasificación en tres grupos:

- creación y fomento de polos empresariales;

- medidas sobre nuevas formas de proporcionar contenidos a los consumidores;

- programas específicos de internacionalización. 
Tabla 1. Valoración de las medidas y su impacto

\begin{tabular}{|l|c|c|l|}
\hline \multicolumn{1}{|c|}{ Clasificación de la medida } & Valoración & Aplicabilidad & \multicolumn{1}{|c|}{ Comentarios } \\
\hline Creación y fomento de polos empresariales & Muy alta & Muy alta & $\begin{array}{l}\text { Esencial para el desarrollo del sector y para crear una industria } \\
\text { de contenidos digitales }\end{array}$ \\
\hline $\begin{array}{l}\text { Búsqueda de nuevas formas de suministrar } \\
\text { contenidos }\end{array}$ & Media & Media & $\begin{array}{l}\text { Las grandes multinacionales de creación de contenidos digita- } \\
\text { les pueden liderar estas medidas }\end{array}$ \\
\hline Programas específicos de internacionalización & Alta & Muy alta & $\begin{array}{l}\text { Necesarios para expandir la industria española en mercados } \\
\text { latinoamericanos }\end{array}$ \\
\hline
\end{tabular}

Para ello se solicitó al mismo grupo de expertos españoles indicado en la fase 0 , que valorasen de manera cualitativa la tipología de las iniciativas y su impacto para la industria española (tabla 1), eligiendo las ocho más relevantes (tabla 2). La investigación se cerró en septiembre de 2013.

A continuación, se describen de forma resumida las medidas finales seleccionadas clasificándolas según la tipología especificada.

Las medidas obtenidas se han clasificado en tres grandes epígrafes: creación y fomento de polos empresariales, medidas sobre nuevas formas de proporcionar contenidos a los consumidores y programas específicos de internacionalización

\subsection{Polos de competitividad}

Las principales iniciativas en este ámbito se han llevado a cabo en el entorno europeo: Alemania, Francia y Reino Unido. En Francia el programa de formación de polos de competitividad (Ministère, 2006) se enmarca dentro de la política económica global francesa desplegada en 2006 para fomentar el crecimiento de aquellos sectores económicos considerados como fuente de trabajo y generadores de empleo. Los polos de competitividad debían canalizar las iniciativas públicas sectoriales y fomentar la colaboración entre empresas del mismo sector.

Para la constitución de los polos se lanzó una convocatoria a la que debían concurrir los posibles organismos gestores de estos polos, en la que se debía presentar un plan de desarrollo. Desde 2009 se han destinado 1.500 millones de eu- ros a este programa y, como resultado del proyecto, 5.000 empresas formaban parte de los polos en 2007 , el $80 \%$ de las cuales eran pequeñas y medianas. Entre ellos destaca el cluster de contenidos digitales denominado Cap Digital, en île de France (alrededores de París), que asocia 20 grandes empresas, más de 600 pequeñas y medianas y 50 institutos de investigación. El cluster incluye varias comunidades: videojuegos, diseño digital, multimedia, noticias culturales, sonido e interactividad, robótica y objetos de comunicación, educación y formación digital, servicios móviles y aplicaciones, ingeniería del conocimiento, software libre y nuevas pautas de cooperación empresarial. De acuerdo con sus propios datos, en la región île de France trabajan 423.000 personas en el sector tecnológico.

En Reino Unido, en noviembre de 2010, el primer ministro David Cameron anunció el compromiso del gobierno de promover en el este de Londres el programa Tech City UK (Tech City, 2011) con objeto de llegar a ser uno de los mayores centros tecnológicos del mundo. La estrategia iba dirigida a apoyar el cluster mediante la creación de las condiciones adecuadas para el crecimiento de los negocios digitales. Como resultado de este compromiso se creó el organismo Tech City Investment Organisation en abril de 2011, dependiente del UK Trade and Investment (agencia de comercio exterior británica), para ayudar al desarrollo del cluster a través de las siguientes actuaciones:

- atrayendo capital extranjero y estableciendo acuerdos de colaboración con empresas de capital riesgo para que se establezcan en Tech City;

- ayudando a los inversores a acceder al flujo de operaciones que se desarrollan en Tech City.

- apoyando a las compañías que quieren expandirse en mercados internacionales (organizando eventos con inversores extranjeros de Europa y Estados Unidos).

Tabla 2. Políticas públicas para la industria de contenidos digitales más relevantes

\begin{tabular}{|c|c|c|c|c|c|}
\hline Clasificación de la medida & Reino Unido & Alemania & Francia & $\begin{array}{l}\text { Estados } \\
\text { Unidos }\end{array}$ & Israel \\
\hline $\begin{array}{l}\text { Creación y fomento de polos } \\
\text { empresariales }\end{array}$ & Tech City UK & $\begin{array}{l}\text { Leading Edge Clus- } \\
\text { ter Competition }\end{array}$ & $\begin{array}{c}\text { Polos de competiti- } \\
\text { vidad }\end{array}$ & & \\
\hline $\begin{array}{l}\text { Búsqueda de nuevas formas de } \\
\text { suministrar contenidos }\end{array}$ & $\begin{array}{c}\text { The Space, Digital Public } \\
\text { Space }\end{array}$ & & & $\begin{array}{l}\text { eTextbooks } \\
\text { educativos }\end{array}$ & \\
\hline $\begin{array}{l}\text { Programas específicos de inter- } \\
\text { nacionalización }\end{array}$ & & $\begin{array}{l}\text { German Silicon } \\
\text { Valley Accelerator }\end{array}$ & & & $\begin{array}{l}\text { Israel Export \& Inter- } \\
\text { national Cooperation } \\
\text { Institute }\end{array}$ \\
\hline
\end{tabular}


Actualmente Tech City alberga más de 200 empresas de contenidos y empresas internacionales de capital riesgo (Solon, 2013). En cuanto al apoyo a la internacionalización de empresas, han organizado más de 25 eventos tanto en Reino Unido como en el extranjero, aunque los análisis de impacto indican que debe potenciar su actividad exportadora.

Dentro de este programa, el gobierno de Reino Unido puso recientemente en marcha el programa Launchpad (UK Innovation, 2013) para fortalecer e impulsar el cluster de contenidos digitales (Tech City, 2012). Su objetivo es continuar nutriendo al cluster de ideas innovadoras y asegurar que se desarrollen en el área proyectos novedosos. Estas ideas o proyectos deben estar orientados a la creación de productos o servicios digitales y están abiertas a compañías creativas.

En Alemania la estrategia digital, tanto a nivel federal como estatal y regional, está orientada al fomento de las agrupaciones empresariales, lo que es común también en otras políticas públicas. En concreto la iniciativa Leading Edge Cluster Competition ha proporcionado asistencia y apoyo financiero a las regiones, estados y empresas para la creación de núcleos empresariales. Lanzada en 2007 , se realizó con el objetivo de fomentar la formación de agrupaciones empresariales a lo largo de Alemania siendo desarrollada por el Ministerio de Educación e Investigación como parte de la estrategia de alta tecnología. Uno de sus principales objetivos es fomentar la creación de puentes entre las empresas, las entidades científicas y las administraciones. A través de tres rondas de financiación se prestó apoyo a quince clusters (con una ayuda financiera pública de hasta 40 millones de euros para cada uno) entre los que se encuentran algunos de los más destacados de la industria digital a nivel europeo.

En la estrategia digital alemana el fomento de las agrupaciones empresariales es común en varias políticas públicas

Uno de los principales contratiempos que las administraciones alemanas han percibido en la industria es el acceso al capital privado, especialmente en las primeras etapas de vida de las empresas, lo que impide que accedan al mercado muchas con formas de negocio prometedoras. Para ello, el gobierno federal y los gobiernos regionales han lanzado una serie de fondos de capital público que aportan financiación a empresas en las primeras etapas hasta que cuentan con madurez suficiente para acceder al capital privado.

\subsection{Nuevas formas de suministrar contenidos}

Las principales iniciativas en este ámbito se han encontrado en Reino Unido y Estados Unidos. En Reino Unido, el proyecto The Space (Arts Council; BBC, 2012) es una alianza entre

la British Broadcasting Corporation (BBC) y el Arts Council of England que tiene como principal objetivo incentivar la experimentación y la innovación en el ámbito de las artes (música, cine, baile, literatura...) y proporcionar una oportunidad de aprender sobre cómo conectar con las audiencias a través de las tecnologías digitales, además de permitir que estas disciplinas alcancen mayores audiencias.

\section{En los EUA el proyecto de libros de texto} educativos electrónicos es un ejemplo en la elaboración de contenidos atractivos en formato digital

The Space proporciona a los artistas una plataforma digital donde mostrar al público sus obras y les brinda la posibilidad de experimentar formas de conectar con la audiencia en un entorno digital muy innovador. Se trata de un programa piloto que estaba inicialmente planificado para el período comprendido entre el 1 de mayo y el 31 de octubre de 2012, prorrogado luego hasta el 31 de marzo de 2013. La plataforma y la infraestructura técnica son gestionadas por la $B B C$ que proporciona también la solución tecnológica.

Otro proyecto es el Digital Public Space (BBC, 2012), cuyo objetivo es compartir el patrimonio cultural del Reino Unido. Nació como una alianza entre la $B B C$ y otras instituciones del país, como museos, archivos, bibliotecas, galerías y organizaciones educativas, que comparten la visión de no utilizar simplemente internet como un canal de distribución de contenidos, sino pasar a formar parte del entorno digital que les rodea.

En Estados Unidos el proyecto de libros de texto educativos electrónicos (Federal Communications Commission, 2012) 


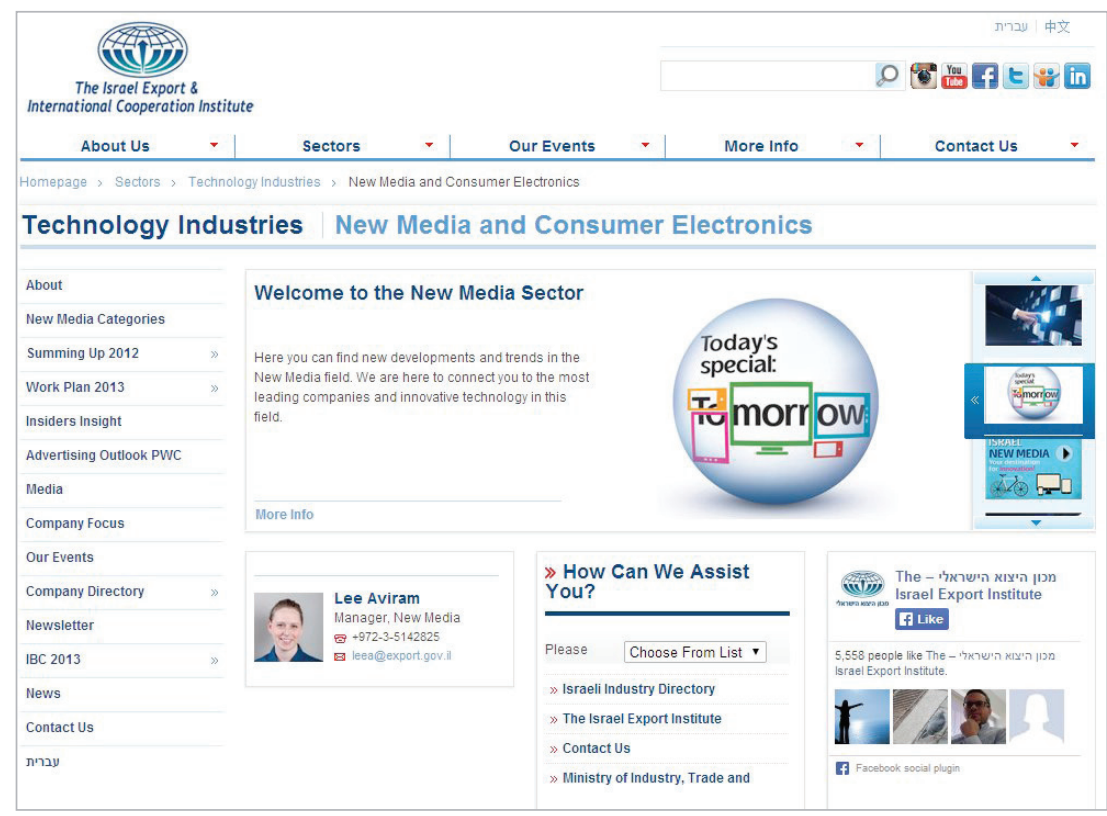

http://www.export.gov.il/eng/Branches/Technologies/Newmedia/.aspx ba. Un ejemplo de ello lo confirma el hecho de que el sector de contenidos digitales en Israel exportó en 2009 una cifra cercana a los 2.000 millones de dólares.

El instituto está estructurado en dos divisiones: bienes de consumo e industria tecnológica. Sobre esta última, el objetivo es incrementar las oportunidades de negocio para las compañías locales, y para ello organiza encuentros de negocios con inversores y grupos de interés extranjeros, ferias y reuniones de negocios, y establece acuerdos de cooperación y colaboración.

En Alemania el German Silicon VaIley Accelerator (Bundesministerium, 2012) tiene su origen en el análisis e intento de superación de tres dificultades que afectan a las nuevas empre-

constituye un ejemplo en el desarrollo de contenidos atractivos en formato digital. El objetivo del gobierno es que todos los estudiantes del país tengan un libro de texto electrónico en 2017. En 2011 en el discurso sobre el estado de la Unión, el presidente Obama dijo: "Quiero que todos los estudiantes puedan aprender a través de dispositivos digitales". La iniciativa, que está siendo liderada por la comisión Leading Education by Advancing Digital, prevé la utilización de libros de texto electrónicos en las escuelas, incluyendo la adquisición de los dispositivos y la tecnología necesaria. La ejecución del plan se está haciendo de forma coordinada con la industria, tanto con empresas tecnológicas como con compañías de telecomunicaciones y agentes del mundo editorial.

La creación de agrupaciones de empresas de contenidos digitales y tecnológicos se ha producido en todos los países relevantes del sector de contenidos

\subsection{Programas de internacionalización}

Las principales iniciativas en el ámbito de la internacionalización se pusieron en marcha en Israel y Alemania. El Israel Export and Internacional Cooperation Institute (leici, 2013) fue creado en 1958 como una organización sin ánimo de lucro mediante un acuerdo entre el sector público y privado. Es el organismo encargado de promocionar las exportaciones de bienes y servicios israelíes, fomentar las relaciones comerciales y establecer alianzas estratégicas y acuerdos de colaboración con compañías extranjeras. El leici nació con un objetivo y una misión clara, superar las limitaciones del mercado local israelí y ayudar a las empresas a expandirse y competir de manera global. La mayor parte de las nuevas empresas en Israel nacen ya con idea de competir en los mercados internacionales utilizando el mercado local israelí a modo de prue- sas tecnológicas alemanas:

- falta de experiencia de los emprendedores alemanes y falta de apoyo profesional;

- mercado europeo heterogéneo;

- limitaciones en la obtención de capital riesgo.

El programa trata de apoyar a las empresas alemanas para que se conviertan en competidores globales, aprovechen las oportunidades financieras que brinda el mercado de Estados Unidos y aceleren su crecimiento aprovechando lo mejor de los dos mercados. La iniciativa proporciona a las empresas seleccionadas un programa acelerador de tres meses en Silicon Valley, pudiéndose prorrogar a otros tres meses adicionales para las compañías más prometedoras. Para determinar las compañías que iban a participar se valoraron más de 80 solicitudes y se seleccionaron diez compañías para el primer programa piloto.

\section{Conclusiones y recomendaciones}

La creación de polos de competitividad sectoriales es una idea llevada a cabo desde los albores de la industria del automóvil, con objeto de ganar en eficiencia, productividad y competitividad. El sector de contenidos no ha sido ajeno y la creación de agrupaciones de empresas de contenidos digitales y tecnológicos se ha producido en todos los países relevantes del sector como Alemania, Francia, Estados Unidos (Silicon Valley, Nueva York), Israel (Tel Aviv), Irlanda (Dublín) e India (Bangalore), entre otros. Los beneficios que proporcionan los polos de competitividad son reconocidos tanto en los aspectos cuantitativos (innovación en productos y servicios, crecimiento económico) como los cualitativos (incremento de la colaboración). Todos los expertos consultados coinciden en que un impulso desde las políticas públicas a la creación de polos de competitividad o clusters sería una medida acertada para el desarrollo del sector de contenidos digitales en España.

La internacionalización en un mercado tan globalizado como el digital puede tener efectos exponenciales en el cre- 
cimiento de las empresas. Uno de los factores fundamentales de las grandes compañías y de los principales proyectos de contenidos es su dimensión internacional. El mejor ejemplo de la importancia de la internacionalización en el sector de contenidos digitales es Israel, donde las limitaciones del mercado local han provocado que las empresas de contenidos israelíes consideren al mercado israelí como un test de aceptación de sus productos, siendo una mera fase previa al mercado mundial.

A juicio de los expertos consultados es necesario generar políticas que ayuden a las empresas TIC españolas y, en particular, a las de contenidos digitales, a posicionarse en el mercado internacional, incluyendo este sector entre las prioridades de las instituciones encargadas de la promoción exterior, aumentando la inteligencia sobre las oportunidades disponibles, facilitando la financiación y las ayudas a la expansión internacional. Para un refuerzo efectivo del sector de los contenidos digitales, España se debe convertir además en un destino atractivo para inversiones TIC de empresas internacionales. Para ello la combinación de políticas fiscales, la simplificación administrativa y la disponibilidad de talento son imprescindibles.

Ejemplos como los de Israel y Estados Unidos han demostrado la importancia de emprender y de obtener capital para el avance de la industria de contenidos digitales. La continua exploración de oportunidades que supone la investigación a lo largo de los diferentes segmentos de la industria digital hace que dos aspectos cobren una gran importancia:

- Disponer de una cultura emprendedora en la sociedad en la que emprender sea concebido como una verdadera alternativa laboral y donde la concepción del fracaso sea la de una forma de aprendizaje.

- Atraer inversores que apoyen a las empresas en sus proyectos a lo largo de las diferentes etapas y necesidades de financiación.

Las políticas orientadas a la creación de nuevos contenidos se consideran también de interés aunque, según los expertos consultados, su aplicabilidad e impacto en la industria española sería menor.

\section{Bibliografía}

Arts Council England; British Broadcasting Corporation (2012). The space.

http://thespace.org/items/e0001n06?t=cwshd

Bouchard, Gerard (2000). Genèse des nations et cultures du nouveau monde. Essai d'histoire comparée. Montreal: Boréal. ISBN: 9782764601105

British Broadcasting Corporation (2012). Digital public space. http://www.bbc.co.uk/blogs/internet/posts/space_arts_projects

Bulcourf, Pablo; Cardozo, Nelson (2008). “Dinámica estatal y políticas públicas: estrategias para la construcción de un modelo de análisis comparado". Nuevo espacio público, n. 1, octubre, pp. 15-54. http://goo.gl/CTUel6

Bundesministerium für wirtschaftliche Zusammenarbeit und Entwicklung (2012). German Silicon Valley accelerator. http://germanaccelerator.com/program/program-overview

Bundesministerium für Bildung und Forschung (2012). Leading edge cluster competition.

http://www.bmbf.de/en/20741.php

Federal Communications Commission; U.S. Department of Education (2012). Digital Textbook Playbook.

http://www.fcc.gov/encyclopedia/digital-textbookplaybook

Gaptel (2006). Contenidos digitales. Nuevos modelos de distribución online. Madrid: Red.es.

http://www.ontsi.red.es/ontsi/sites/default/files/3_2_0.pdf

Grau, Mireia; Mateos, Araceli (2002). Análisis de políticas públicas en España: enfoques y casos. Valencia: Tirant lo Blanch. ISBN: 9788484426622

Israel Exports and Internacional Cooperation Institute (2013). New media.

http://www.export.gov.il/eng/Branches/Technologies/ Newmedia/.aspx

Ministère de l'Economie et des Finances (2006). Les pôles de compétitivité.

http://competitivite.gouv.fr

Ministerio de Industria, Energía y Turismo (2013a). Agenda digital para España.

http://www.agendadigital.gob.es/agenda-digital/recursos/ Recursos/1.\%20Versi\%C3\%B3n\%20definitiva/Agenda_ Digital_para_Espana.pdf

Ministerio de Industria, Energía y Turismo (2013b). Plan de impulso de la economía digital y los contenidos digitales.

http://www.agendadigital.gob.es/planes-actuaciones/ Paginas/plan-impulso-contenidos-digitales.aspx

Ontsi (2012). Informe anual de los contenidos digitales en España (edición 2012).

http://goo.gl/9hJJsE

Parsons, Wayne (2008). Políticas públicas: una introducción a la teoría y la práctica del análisis de las políticas públicas. Buenos Aires: Miño y Dávila. ISBN: 9789709967067

Smelser, Neil J. (2003). "On comparative analysis, interdisciplinarity and internationalization in sociology". International sociology, v. 18, n. 4, pp. 643-658.

http://goo.gl/iL5pGg

Solon, Olivia (2013). "Private sector advocacy group aims to support London tech scene". Wired magazine.

http://www.wired.co.uk/news/archive/2013-04/17/techlondon-advocates

Tech City (2011). Tech City impact report. http://brandfeed.co/brand/post/view/164/13170

UK Innovation Agency (2013). Launchpads. http://goo.gl/S103JT

Urueña-López, Alberto; Hidalgo-Nuchera, Antonio (2013). "La información en la economía del conocimiento: retos y oportunidades para España". El profesional de la información, julio-agosto, v. 22, n. 4, pp. 339-345. http://dx.doi.org/10.3145/epi.2013.jul.10 